\title{
Uma revisão sistemática da literatura: análise sobre desigualdade estrutural em decorrência de casos de dengue e sua influência no cenário brasileiro
}

A systematic literature review: analysis of structural inequality due to dengue cases and its

\author{
influence on the Brazilian scenario
}

Una revisión sistemática de la literatura: análisis de la desigualdad estructural por casos de dengue y su influencia en el escenario brasileño

Recebido: 12/08/2021 | Revisado: 16/08/2021 | Aceito: 21/08/2021 | Publicado: 23/08/2021

\author{
Mônica dos Santos Alves \\ ORCID: https://orcid.org/0000-0002-9848-487X \\ Universidade Estadual da Paraíba, Brasil \\ E-mail: Monica23081997@ @otmail.com \\ Wellington Candeia de Araújo \\ ORCID: https://orcid.org/0000-0003-2102-7993 \\ Universidade Estadual da Paraíba, Brasil \\ E-mail: wcandeia@gmail.com \\ Francisco Anderson Mariano da Silva \\ ORCID: https://orcid.org/0000-0001-9797-7552 \\ Universidade Estadual da Paraíba, Brasil \\ E-mail: Franciscoanderson4@gmail.com
}

\begin{abstract}
Resumo
No tempo atual, a grande incidência de dengue tem relação com a desigualdade estrutural e da falta de saneamento básico encontrados em vários ambientes precários, na qual residem famílias em situações suscetíveis. Este trabalho tem como objetivo analisar a ocorrência de casos por dengue no período de 2015 a 2020, assim como determinar uma associação de como os fatores socioeconômicos como moradias inadequadas, renda mínima, aglomerações de famílias, densidades domiciliar e a inexistência de saneamento básico, têm potencial de influir na propagação da doença. Este estudo teve como finalidade expor uma revisão sistemática de trabalhos acadêmicos, anteriormente publicados sobre o enfoque da dengue na vida das pessoas e o perigo que o mesmo oferece à população. Os resultados encontrados apresentaram incidência positiva na correlação com os fatores sociais citados, favorecendo assim a proliferação do Aedes aegypti.
\end{abstract}

Palavras-chave: Influência; Desigualdade estrutural; Casos por Dengue; Regiões precárias.

\begin{abstract}
Currently, the high incidence of dengue is related to structural inequality and the lack of basic sanitation found in various precarious environments, in which families live in susceptible situations. This work aims to analyze the occurrence of dengue cases in the period from 2015 to 2020, as well as to determine an association of how socioeconomic factors such as inadequate housing, minimum income, family agglomerations, household densities and the lack of basic sanitation, have potential to influence the spread of the disease. This study aimed to present a systematic review of previously published academic papers on the focus of dengue in people's lives and the danger it poses to the population. The results found showed a positive incidence in the correlation with the social factors mentioned, thus favoring the proliferation of Aedes aegypti.
\end{abstract}

Keywords: Influence; Structural inequality; Dengue cases; Precarious regions.

\section{Resumen}

Actualmente, la alta incidencia del dengue está relacionada con la desigualdad estructural y la falta de saneamiento básico que se encuentra en diversos entornos precarios, en los que las familias viven en situaciones susceptibles. Este trabajo tiene como objetivo analizar la ocurrencia de casos de dengue en el período de 2015 a 2020, así como determinar una asociación de cómo factores socioeconómicos como vivienda inadecuada, ingresos mínimos, aglomeraciones familiares, densidades de hogares y la falta de saneamiento básico, han potencial para influir en la propagación de la enfermedad. Este estudio tuvo como objetivo presentar una revisión sistemática de trabajos académicos publicados anteriormente sobre el enfoque del dengue en la vida de las personas y el peligro que representa para la población. Los resultados encontrados mostraron una incidencia positiva en la correlación con los factores sociales mencionados, favoreciendo así la proliferación de Aedes aegypti.

Palabras clave: Influencia; Desigualdad estructural; Casos de dengue; Regiones precarias. 


\section{Introdução}

Arboviroses são enfermidades provocadas por arbovírus, que engloba os vírus da zika, dengue, febre chikungunya e febre amarela, todas com extensa movimentação pelo território brasileiro e são transmitidas pelo Aedes aegypti. De acordo com Machado, Oliveira e Souza-Silva (2009, p.1025) a dengue refere-se, consequentemente, a um preocupante problema de bemestar público, especialmente em países que passam por situações socioambientais, socioeconômicas e climáticas que facilitam a evolução e a propagação do vetor intermediário.

De acordo com o Ministério da Saúde (2020), o vetor da dengue necessita de água parada para se proliferar. A estação do ano com maior transmissão é os dias chuvosos de cada local, porém é relevante manter a limpeza e evitar água parada todos os dias, posto que, os ovos do mosquito conseguem sobreviver por um ano até se deparar com melhores circunstâncias para se desenvolver. Por esse motivo, é significativo evitar acúmulo de água em vasilhames como garrafas e pneus, vasos de plantas, e permanecer com os reservatórios de água fechados.

Refutam-se na literatura que a propagação das arboviroses sucede em sua maior porção pelo contato com o agente transmissor, a mais adequada forma de precaução de arboviroses é exatamente safando-se do contato.

Mendonça, Souza e Dutra (2009, p.259) aponta que mesmo diante de um grande problema de saúde pública, a melhor forma de entender a gravidade e a ocorrência de casos por dengue, zika e chikungunya, é buscando analisar qual indicador social está servindo de abrigo para o mosquito Aedes aegypti, facilitando assim a propagação.

Estudos relatam que a maioria dos focos se encontra nas residências, na debilidade da limpeza pública, na acumulação de resíduos sólidos urbanos, nas falhas de esgoto sanitário pelos sistemas públicos, além do agrupamento populacional de pessoas suscetíveis, são estes os aspectos que possibilitam a propagação do vetor. Nesse sentido, pessoas que vivem em moradias com condições inadequadas, em regiões suburbanas, em aglomerações subnormais, renda baixa e sem saneamento básico, estão sujeitas a maior perigo de exposição e proliferação do transmissor.

Diante do exposto, o objetivo geral desta revisão é analisar, por meio de publicações científicas, estudos já realizados sobre a associação da dengue com a desigualdade estrutural, do ponto de vista de pesquisadores, no contexto social com foco em estudos. Destaca-se que para a realização desta revisão foram construídas duas questões/problemas: Q1 - Quais estudos com foco na influência que a desigualdade estrutural tem sobre a saúde e sobre a frequência de casos por dengue foram feitos até o momento? Q2 - Dentre os estudos encontrados, como esse indicador social influencia na proliferação e na propagação da dengue em ambientes sem saneamento básico?

\section{Metodologia}

A revisão sistemática é o estudo científico que busca agrupar publicações relevantes acerca de uma pergunta elaborada, que será buscada em banco de dados e em seguida, analisada de forma crítica e cautelosa, a fim de que, seja realizada uma revisão precisa e importante sobre o tema proposto.

Linde e Willich (2002, p.17) dizem que, procedimentos sistemáticos são planejados para desviar-se de vieses e transformar as consequências e finalizações o mais objetivo realizável. Entretanto, as revisões sistemáticas são consideradas retrospectos e necessitam vigorosamente de excelente material antecedente.

Foi executada a revisão sistemática em três fases: o planejamento, a execução da pesquisa e a análise dos resultados adquiridos, que serão detalhados seguidamente. 


\subsection{Planejamento}

De acordo com Hulley et al. (2003, p.21) a base de um projeto de pesquisa é retratada em seu protocolo, o esboço redigido do aprendizado. O protocolo é uma ferramenta utilizada na requisição de meios monetários, porém assim como possui mais um papel científico essencial, que é o auxílio para o pesquisador metodizar a sua pesquisa de modo coerente, direto e ágil.

\subsection{Definição das questões de pesquisa}

- Q1 - Quais estudos com foco na influência que a desigualdade estrutural tem sobre a saúde e sobre a frequência de casos por dengue foram feitos até o momento?

- Q2 - Dentre os estudos encontrados, como esse indicador social influencia na proliferação e na propagação da dengue em ambientes sem saneamento básico?

\subsection{Seleção de fontes}

Foram aplicadas expressões de busca na procura por procedências bibliográficas através da Internet, a fim de conseguir versões digitais de periódicos. Caso assim não seja realizável conseguir publicações completas por meio das páginas da internet de busca, serão contatados via e-mail os autores das publicações.

\subsection{Idiomas}

Os idiomas escolhidos foram o inglês e o português, por serem os mais adotados em conferências e periódicos nacionais e internacionais relacionados com o tema de pesquisa.

\subsection{String de Busca}

Para buscas por produções em português é preciso empregar a expressão seguinte:

- "Influência" AND "Desigualdade" AND "Casos" AND "Dengue".

Para buscas por produções em inglês é preciso empregar a expressão seguinte:

- "Influence" AND "Inequality" AND "Cases" AND "Dengue".

A string foi aplicada no Portal de Periódicos da CAPES, na PubMed e na Scopus, retornando um total de artigos satisfatórios.

\subsection{Restrições}

A pesquisa está restrita à investigação de trabalhos realizados, com fundamentação das fontes selecionadas, no período de 18 de janeiro de 2015 até 31 de dezembro de 2020.

\subsection{Critérios de Inclusão}

Os critérios que serão levados em conta para a Inclusão:

- CI1. Serão escolhidas publicações que contêm o termo Desigualdade e Dengue.

- CI2. Serão escolhidas publicações com foco na influência que a desigualdade estrutural tem sobre a saúde e a ocorrência de casos por dengue.

- CI3. Artigos 2015-2020. 


\subsection{Critérios de Exclusão}

Os critérios de Exclusão serão:

- CE1. Não serão escolhidas publicações que não tenham nenhuma relevância com os critérios

- de inclusão

- CE2. Não serão escolhidas publicações que não estejam nas línguas de português e inglês;

- CE3. Não serão escolhidas publicações que não trazem os termos Desigualdade e Dengue;

- CE4. Não serão escolhidas publicações que não tem foco na influência que a desigualdade estrutural tem sobre a saúde e a ocorrência de casos por dengue;

- CE5. Serão excluídas revisões sistemáticas, tendo em vista que estamos realizando um estudo primário.

\subsection{Período de Busca}

O período de busca foi delimitado de 18 de janeiro de 2015 até 31 de dezembro de 2020.

\section{Resultados e Discussão}

Ao ser inserida a expressão de busca com os termos Desigualdade e Dengue nas bases indexadas, foram identificados um total de 675 resultados. Desse retorno, apenas 27 artigos apresentavam relação entre desigualdade e dengue. Com base em uma leitura minuciosa dos títulos, abstracts e aplicação dos critérios de inclusão e exclusão, 15 artigos trouxeram a relação da desigualdade estrutural com a saúde e a ocorrência de casos por dengue.

No Quadro 1, estão expostos os 15 artigos que foram identificados através da string: "influência" da "desigualdade" em “casos" por “dengue” em "regiões precárias” no período de "2015 até 2020”, organizados em uma sequência numérica, por títulos dos seus respectivos artigos, endereços de onde foram encontrados, seus devidos autores, a base de dados que publicaram o artigo e o ano de publicação.

Quadro 1 - Artigos selecionados.

\begin{tabular}{|c|c|c|c|c|c|}
\hline ID & Título & Endereço & Autores & $\begin{array}{c}\text { Base de } \\
\text { Dados }\end{array}$ & Ano \\
\hline A1 & $\begin{array}{l}\text { Aedes Mosquito Infestation in } \\
\text { Socioeconomically Contrasting } \\
\text { Neighborhoods of Panama City }\end{array}$ & $\begin{array}{l}\text { https://link.springer.co } \\
\text { m/article/10.1007/s10 } \\
\text { 393-019-01417-3 }\end{array}$ & $\begin{array}{l}\text { Ari Whiteman, Carmelo Gomez, } \\
\text { Jose Rovira, Gang Chen, W. Owen } \\
\text { McMillan \& Jose Loaiza }\end{array}$ & Springer & 2019 \\
\hline A2 & $\begin{array}{l}\text { A participatory community case } \\
\text { study of periurban coastal flood } \\
\text { vulnerability in southern Ecuador }\end{array}$ & $\begin{array}{l}\text { https://journals.plos.or } \\
\text { g/plosone/article?id=1 } \\
0.1371 \text { /journal.pone. } 0 \\
\text { 224171 }\end{array}$ & $\begin{array}{c}\text { Erica Tauzer, Mercy J Borbor- } \\
\text { Cordova, Jhoyzett Mendoza, Telmo } \\
\text { De La Cuadra, Jorge Cunalata, } \\
\text { Anna M Stewart-Ibarra }\end{array}$ & Journals Plos & 2019 \\
\hline $\mathrm{A} 3$ & $\begin{array}{l}\text { A Perspective on Inhabited Urban } \\
\text { Space: Land Use and Occupation, } \\
\text { Heat Islands, and Precarious } \\
\text { Urbanization as Determinants of } \\
\text { Territorial Receptivity to Dengue } \\
\text { in the City of Rio De Janeiro }\end{array}$ & $\begin{array}{c}\text { https://www.mdpi.co } \\
\text { m/1660- } \\
4601 / 17 / 18 / 6537\end{array}$ & $\begin{array}{c}\text { Jefferson Pereira Caldas Santos, } \\
\text { Nildimar Alves Honório, } \\
\text { Christovam Barcellos and Aline } \\
\text { Araújo Nobre }\end{array}$ & MDPI & 2020 \\
\hline A4 & $\begin{array}{l}\text { Determining the association } \\
\text { between dengue and social } \\
\text { inequality factors in north-eastern } \\
\text { Brazil: A spatial modelling }\end{array}$ & $\begin{array}{l}\text { https://www.researchg } \\
\text { ate.net/publication/34 } \\
\text { 2274794_Determining } \\
\text { _the_association_betw } \\
\text { een_dengue_and_soci } \\
\text { al_inequality_factors_ }\end{array}$ & $\begin{array}{l}\text { Damião Da Conceição Araújo, } \\
\text { Allan Dantas dos Santos, Shirley } \\
\text { Verônica Melo Almeida Lima, } \\
\text { Andreia Centenaro Vaez, Jéssica } \\
\text { Oliveira da Cunha, Karina }\end{array}$ & ResearchGate & 2020 \\
\hline
\end{tabular}




\begin{tabular}{|c|c|c|c|c|c|}
\hline & & $\begin{array}{l}\text { in_north- } \\
\text { eastern_Brazil_A_spat } \\
\text { ial_modelling }\end{array}$ & $\begin{array}{c}\text { Conceição Gomes Machado de } \\
\text { Araujo }\end{array}$ & & \\
\hline A5 & $\begin{array}{l}\text { Gestão adequada de resíduos } \\
\text { sólidos como fator de proteção na } \\
\text { ocorrência da dengue }\end{array}$ & $\begin{array}{l}\text { https://scielosp.org/art } \\
\text { icle/rpsp/2020.v44/e2 } \\
\text { 2/ }\end{array}$ & $\begin{array}{c}\text { Marcos Paulo Gomes Mol, Josiane } \\
\text { T. Matos Queiroz, Júlia Gomes, Léo } \\
\text { Heller }\end{array}$ & Scielosp & 2020 \\
\hline A6 & $\begin{array}{l}\text { Sanitation, Arboviruses, and } \\
\text { Environmental Determinants } \\
\text { of Disease: impacts on urban } \\
\text { health }\end{array}$ & $\begin{array}{l}\text { https://www.scielosp. } \\
\text { org/pdf/csc/2020.v25n } \\
\text { 10/3857-3868/pt }\end{array}$ & $\begin{array}{c}\text { Lorena Sampaio Almeida, Ana } \\
\text { Lídia Soares Cota, Diego Freitas } \\
\text { Rodrigues }\end{array}$ & Scielosp & 2020 \\
\hline A7 & $\begin{array}{l}\text { Socio-economic and Climate } \\
\text { Factors Associated with Dengue } \\
\text { Fever Spatial Heterogeneity: A } \\
\text { Worked Example in New } \\
\text { Caledonia }\end{array}$ & $\begin{array}{c}\text { https://journals.plos.or } \\
\text { g/plosntds/article?id= } \\
\text { 10.1371/journal.pntd. } \\
\text { 0004211 }\end{array}$ & $\begin{array}{l}\text { Magali Teurlai, Christophe Eugène } \\
\text { Menkès, Virgil Cavarero, Nicolas } \\
\text { Degallier, Elodie Descloux, Jean- } \\
\text { Paul Grangeon, Laurent Guillaumot, } \\
\text { Thérèse Libourel, Paulo Sergio } \\
\text { Lucio, Françoise Mathieu-Daudé, } \\
\text { Morgan Mangeas }\end{array}$ & Journals Plos & 2015 \\
\hline A8 & $\begin{array}{l}\text { Spatial Distribution of Dengue in a } \\
\text { Brazilian Urban Slum Setting: } \\
\text { Role of Socioeconomic Gradient } \\
\text { in Disease Risk }\end{array}$ & $\begin{array}{l}\text { https://journals.plos.or } \\
\text { g/plosntds/article?id= } \\
\text { 10.1371/journal.pntd. } \\
\text { 0003937\#abstract1 }\end{array}$ & $\begin{array}{l}\text { Mariana Kikuti, Geraldo M. Cunha, } \\
\text { Igor A. D. Paploski, Amelia M. } \\
\text { Kasper, Monaise M. O. Silva, Aline } \\
\text { S. Tavares, Jaqueline S. Cruz, } \\
\text { Tássia L. Queiroz, Moreno S. } \\
\text { Rodrigues, Perla M. Santana, } \\
\text { Helena C. A. V. Lima, Juan } \\
\text { Calcagno, Daniele Takahashi, } \\
\text { André H. O. Gonçalves, Josélio M. } \\
\text { G. Araújo, Kristine Gauthier, Maria } \\
\text { A. Diuk-Wasser, Uriel Kitron, } \\
\text { Albert I. Ko, Mitermayer G. Reis, } \\
\text { Guilherme S. Ribeiro }\end{array}$ & Journals Plos & 2015 \\
\hline A9 & $\begin{array}{l}\text { Spatiotemporal distribution and } \\
\text { socioeconomic disparities of } \\
\text { dengue, chikungunya and Zika in } \\
\text { two Latin American cities from } \\
2007 \text { to } 2017\end{array}$ & $\begin{array}{l}\text { https://pubmed.ncbi.nl } \\
\text { m.nih.gov/33219561/ }\end{array}$ & $\begin{array}{c}\text { Mabel Carabali, Sam Harper, } \\
\text { Antonio S Lima Neto, Geziel Dos } \\
\text { Santos de Sousa, Andrea Caprara, } \\
\text { Berta Nelly Restrepo, Jay S } \\
\text { Kaufman }\end{array}$ & PubMed & 2020 \\
\hline A10 & $\begin{array}{l}\text { Spatiotemporal dynamics, risk } \\
\text { areas and social determinants of } \\
\text { dengue in Northeastern Brazil, } \\
\text { 2014-2017: an ecological study }\end{array}$ & $\begin{array}{c}\text { https://idpjournal.bio } \\
\text { medcentral.com/articl } \\
\text { es/10.1186/s40249- } \\
\text { 020-00772-6 }\end{array}$ & $\begin{array}{c}\text { Rodrigo Feliciano do Carmo, José } \\
\text { Valter Joaquim Silva Júnior, Andre } \\
\text { Filipe Pastor \& Carlos Dornels } \\
\text { Freire de souza }\end{array}$ & $\mathrm{BMC}$ & 2020 \\
\hline A11 & $\begin{array}{l}\text { The COVID-19 pandemic should } \\
\text { not jeopardize dengue control }\end{array}$ & $\begin{array}{c}\text { https://www.researchg } \\
\text { ate.net/publication/34 } \\
4367585 \text { The_COVI } \\
\text { D- } \\
\text { 19_pandemic_should_ } \\
\text { not_jeopardize_dengu } \\
\text { e_control }\end{array}$ & $\begin{array}{c}\text { Marie-Marie Olive, Thierry Baldet, } \\
\text { James Devillers, Johanna Fite, } \\
\text { Marie-Claire Paty, Christophe } \\
\text { Paupy, Philippe Quénel, Elsa } \\
\text { Quillery, Jocelyn Raude, Jean-Paul } \\
\text { Stahl, Marie Thiann-Bo-Morel, } \\
\text { David Roiz }\end{array}$ & ResearchGate & 2020 \\
\hline A12 & $\begin{array}{l}\text { The social face of Aedes control: } \\
\text { women take the floor in a } \\
\text { suburban district of Fortaleza, } \\
\text { Brazil }\end{array}$ & $\begin{array}{l}\text { https://scielosp.org/art } \\
\text { icle/csc/2019.v24n8/2 } \\
\text { 983-2992/en/ }\end{array}$ & $\begin{array}{c}\text { Krysne Kelly de França Oliveira, } \\
\text { Andrea Caprara }\end{array}$ & Scielosp & 2019 \\
\hline A13 & $\begin{array}{c}\text { Trends and factors associated with } \\
\text { dengue mortality and fatality in } \\
\text { Brazil }\end{array}$ & $\begin{array}{l}\text { https://www.scielo.br/ } \\
\text { scielo.php?script=sci_ } \\
\text { arttext\&pid=S0037- } \\
86822015000400399\end{array}$ & $\begin{array}{c}\text { Enny Santos Paixão, Maria da } \\
\text { Conceição Nascimento Costa, Laura } \\
\text { Cunha Rodrigues, Davide Rasella, } \\
\text { Luciana Lobato Cardim, Alcione } \\
\text { Cunha Brasileiro, Maria Gloria } \\
\text { Lima Cruz Teixeira }\end{array}$ & Scielo.br & 2015 \\
\hline
\end{tabular}




\begin{tabular}{|c|c|c|c|c|c|}
\hline A14 & $\begin{array}{c}\text { Vulnerability, health and disasters } \\
\text { in São Paulo coast (Brazil): } \\
\text { challenges for a sustainable } \\
\text { development }\end{array}$ & $\begin{array}{c}\text { https://www.scielo.br/ } \\
\text { scielo.php?script=sci_- } \\
\text { arttext\&pid=S1414- } \\
753 X 2018000100403\end{array}$ & $\begin{array}{c}\text { Luciana de Resende Londe, Lívia } \\
\text { Gonzaga Moura, Marcos Pellegrini } \\
\text { Coutinho, Victor Marchezini, Erico } \\
\text { Soriano }\end{array}$ & Scielo.br & 2018 \\
\hline A15 & $\begin{array}{c}\text { Who are they, what do they talk } \\
\text { about and who listens to the poor? }\end{array}$ & $\begin{array}{c}\text { https://www.scielo.br/ } \\
\text { scielo.php?script=sci_ } \\
\text { arttext\&pid=S1413- } \\
81232017021203859\end{array}$ & $\begin{array}{c}\text { Maraiza Alves Freitas, Augustus } \\
\text { Tadeu Relo de Mattos, William } \\
\text { Zaccaro Gomes, Maria do Carmo } \\
\text { Gullaci Guimarães Caccia-Bava }\end{array}$ & Scielo.br & 2017 \\
\hline
\end{tabular}

Fonte: Autores (2021).

No tocante à questão de pesquisa Q1, foram encontrados inúmeros estudos que traziam a influência que a desigualdade estrutural causa à saúde pública e na frequência de casos por dengue de forma clara. Entretanto, no propósito de responder às duas questões de pesquisa, os 15 artigos selecionados trouxeram todo o amparo necessário para a realização da pesquisa, o conhecimento primordial e o fundamento preciso para responder de forma adequada às questões de pesquisa do estudo. Com base nos artigos lidos, foi trazido todo o conteúdo absorvido e que será apresentado logo a seguir, abordando a desigualdade estrutural, a saúde e a frequência de casos por dengue.

O crescimento da população mundial sem preparação e a urbanização sem o devido planejamento e controle, principalmente nos países que estão se desenvolvendo, estão associados ao ressurgimento da dengue (Gubler, 1998, p.482).

Com a crescente quantidade de pessoas vivendo na zona urbana no decorrer dos séculos XIX e XX, os municípios transformaram-se em focos de aglutinação de pobreza, deslocação social e infração (Ribeiro; Vargas, 2015).

De acordo com a análise feita por Alves (2021, p. 9), é inquestionável que no decorrer do período de 2015 a 2020 , vários estados do território brasileiro tiveram aumento ou diminuição dos seus casos por dengue. Classificada como a área mais abundante e povoada do brasil, a região Sudeste vem comandando os topos na ocorrência de casos. Logo depois apresenta-se a região Nordeste, a área mais carente do país, onde a maior parte encontra-se em um ambiente semiárido. Em seguida aparece a região Centro-Oeste, que é uma área com farto controle agrícola. Adiante está a região Sul, que é caracterizada por ter uma temporada mais equilibrada. E finalmente vem a região Norte, no qual a sua maior parte territorial é ocupada por floresta tropical.

O Quadro 2, contém os dados sobre os rendimentos domiciliar per capita médio, por classes de percentual de domicílios em ordem crescente de rendimento, com indicação do coeficiente de variação no Brasil em 2019, disponibilizados pelo Instituto Brasileiro de Geografia e Estatística (IBGE):

Quadro 2 - Dados de rendimento domiciliar per capita médio.

\begin{tabular}{|c|c|c|c|c|c|c|c|c|}
\hline & \multicolumn{6}{|c|}{ Rendimento domiciliar per capita médio das pessoas (R\$) } \\
\cline { 2 - 8 } $\begin{array}{c}\text { Grandes } \\
\text { Regiões, } \\
\text { Unidades da } \\
\begin{array}{c}\text { Federação } \\
\text { Municípios } \\
\text { das Capitais }\end{array}\end{array}$ & $\begin{array}{c}\text { 40\% com menores } \\
\text { rendimentos (A) }\end{array}$ & $\begin{array}{c}10 \% \text { com maiores } \\
\text { rendimentos (B) }\end{array}$ & $\begin{array}{c}\text { 20\% com menores } \\
\text { rendimentos (C) }\end{array}$ & $\begin{array}{c}\text { 20\% com maiores } \\
\text { rendimentos (D) }\end{array}$ \\
\cline { 2 - 8 } & Valor & $\mathrm{CV}(\%)$ & Valor & $\mathrm{CV}(\%)$ & Valor & $\begin{array}{c}\text { CV } \\
(\%)\end{array}$ & Valor & CV (\%) \\
\hline Brasil & $\mathbf{3 5 8}$ & $\mathbf{0 , 4}$ & $\mathbf{6 0 2 7}$ & $\mathbf{1 , 7}$ & $\mathbf{2 0 4}$ & $\mathbf{0 , 5}$ & $\mathbf{4 1 1 0}$ & $\mathbf{1 , 5}$ \\
\hline
\end{tabular}

Fonte: Dados do IBGE (2019). 
No Brasil, os grandes estados em circunstâncias populacionais, são os que mais apresentaram maior crescimento econômico e público. Nota-se que os grandes estados que possuem posições máximas de pib per capita e que dispõem de mais mecanismos e progresso, foram os que mais viveram com a frequência da enfermidade. (Alves, 2021).

Os indivíduos mais ricos conduzem-se a sair dos perigos que o ambiente proporciona, dirigindo-se para regiões mais protegidas. Já os indivíduos mais pobres, são impulsionados para situações degradadas por um mecanismo dobrado (Acselrad; Mello; Bezerra, 2008, p.78). Nesse mecanismo dobrado, as pessoas de menor renda que são impulsionadas para situações de maior perigo e com não muita infraestrutura, como da mesma forma as fontes de ameaça ambiental e de enorme colisão ecológica são também conduzidas para essas áreas.

De acordo com Caiaffa et al. (2007, p.341) os fatores consideravelmente relacionados aos lugares de maior risco foram renda mínima do líder da família, maior condensação familiar e maior dimensão de pessoas idosas e crianças.

No Brasil temos a Constituição de 1988 que nos beneficia com o direito à moradia (Brasil, 2015), temos também uma sequência de programas de leis do governo voltados para esse propósito, entre eles podemos citar o Plano Nacional de Habitação (PlanHab) e o Programa de Aceleração do Crescimento (PAC), mesmo com essas cláusulas ao nosso dispor, perdura até agora uma carência habitacional calculado em aproximadamente 7,9 milhões de residências no Brasil (IPEA, 2009). O ingresso a um lar apropriado não é a real vivência de muitas pessoas.

Gráfico 1 - Dados de restrição a condições de moradia.

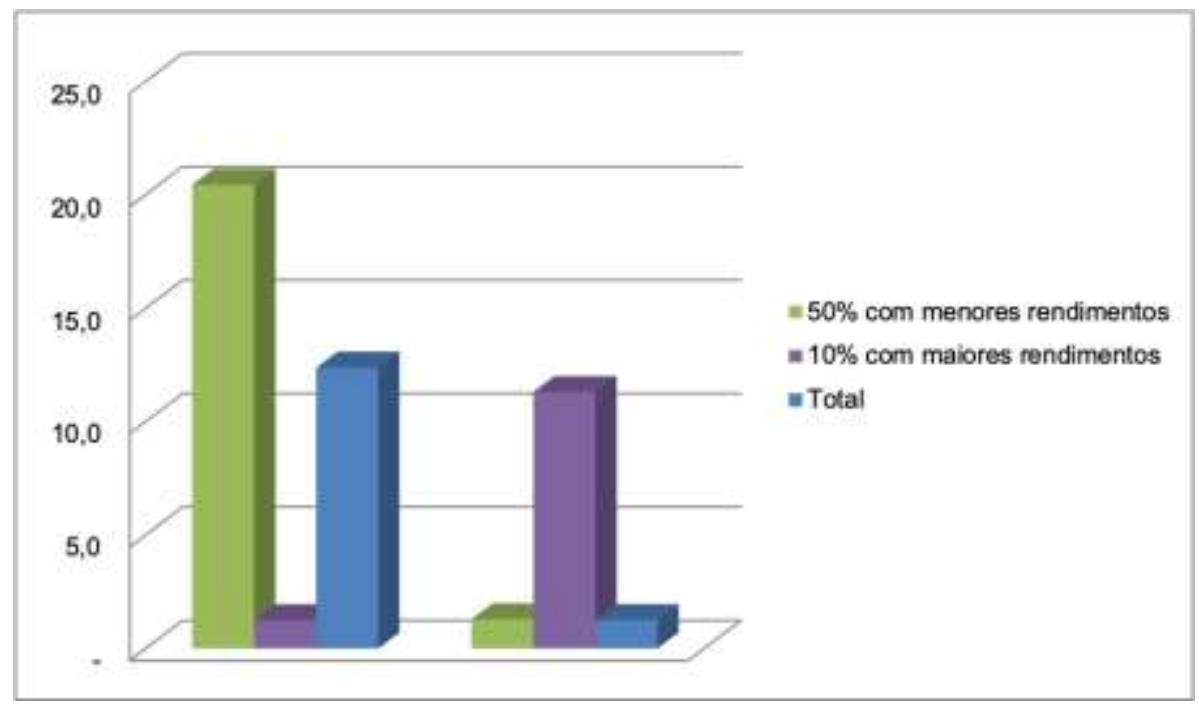

Fonte: Autores (2021).

No Gráfico 1, com os dados obtidos pelo IBGE, podemos observar que 50\% das pessoas com menores rendimentos têm restrições a condições de moradia, enquanto que $10 \%$ das pessoas com maiores rendimentos têm a proporção menor se tratando de restrição à moradia.

O gráfico seguinte, Gráfico 2, traz os dados referentes às inadequações nas condições de moradia que são, respectivamente codificados: IN1-ausência de banheiro de uso exclusivo do domicílio, IN2-paredes externas construídas predominantemente com materiais não duráveis, IN3-adensamento excessivo, IN4-ônus excessivo com aluguel, IN5-ausência de documento que comprove a propriedade e IN6-ao menos uma inadequação nas condições de moradia; dados baseados na vida dos brasileiros e disponibilizados pelo IBGE: 
Gráfico 2 - Dados de inadequações de moradia.

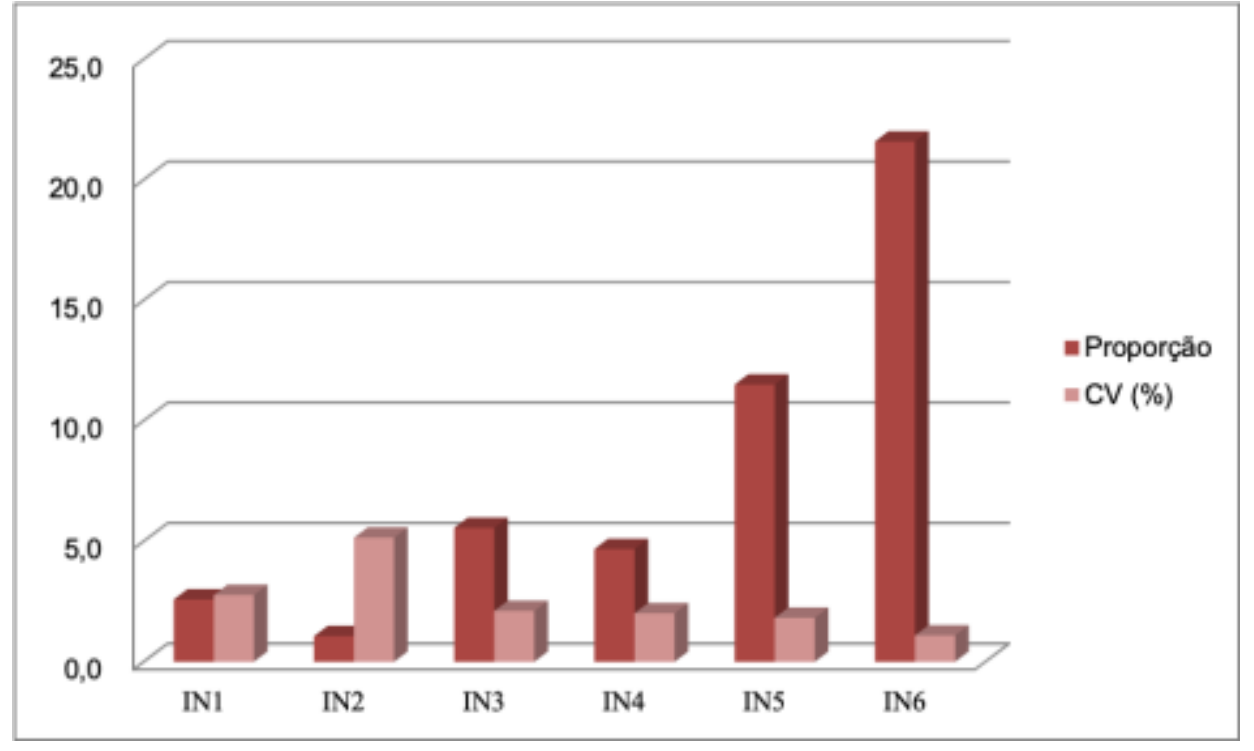

Fonte: Autores (2021).

Segundo Barcellos et al., (2002, p.130), a enfermidade pode ser encarada como uma revelação individual, no entanto, o cenário de saúde é uma exteriorização do espaço em que habita e vive.

De acordo com Sousa Sousa e Alvares (2015, p.104), o saneamento básico é uma agregação de regras para preservação do ecossistema e precaução de enfermidades, em outras palavras, é uma agregação de interferências multidimensionais estruturadas a motivos políticos, econômicos, sociais e culturais que integram os conjuntos de abastecimento de água, a drenagem de água pluviais, a limpeza urbana, o esgotamento sanitário e outros conjuntos.

Nos gráficos a seguir (Gráficos 3 e 4), mostram-se a proporção de brasileiros que têm restrição a serviços de saneamento básico e de modo geral, as características de saneamento do domicílio em que os mesmos vivem:

Gráfico 3 - Dados de restrição a serviços de saneamento básico.

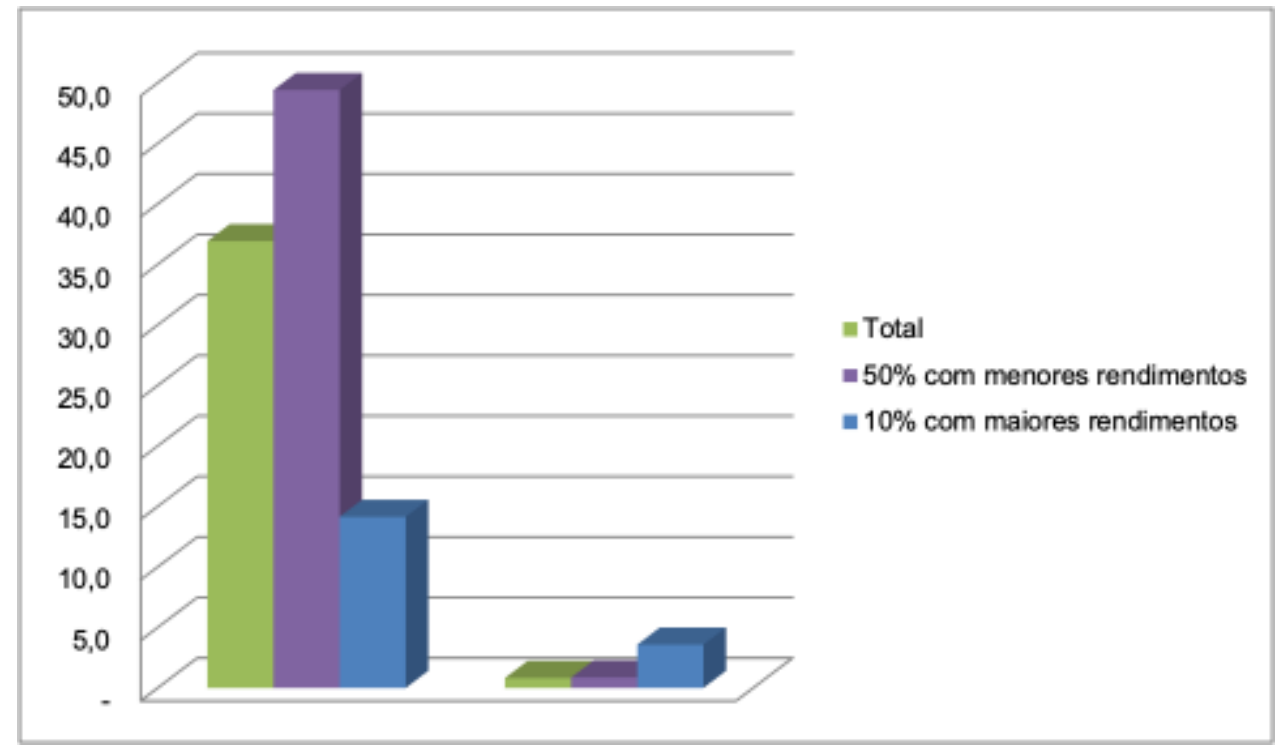

Fonte: Autores (2021). 
Gráfico 4 - Dados das características de saneamento do domicílio.

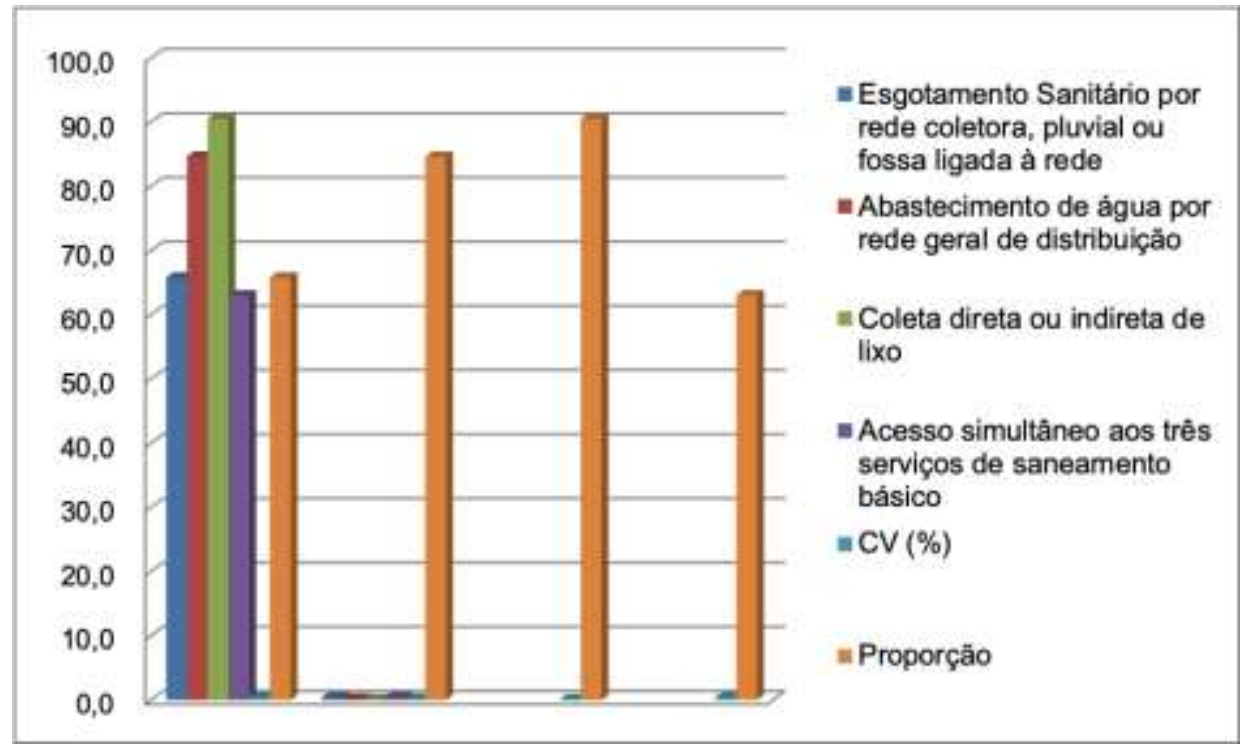

Fonte: Autores (2021).

A inexistência de saneamento básico ou a incapacidade na oferta dessas comodidades, especialmente no que se refere a particularidade da água ofertada para o abastecimento familiar e das qualidades dos rejeitos jogados em corpos d'água coopera expressivamente para a debilidade do bem-estar público de um local (Follador et al., 2015, p.24).

Com relação à questão de pesquisa Q2, os estudos encontrados nos conduziram a ligação que o indicador propicia para que a dengue em lugares sem saneamento básico venha a se proliferar e se propagar, tornando assim, um ambiente de risco para as pessoas que ali vivem.

Tauil (2001, p.99-102), menciona que o crescimento populacional, a urbanização inapropriada, o duro exercício dos sistemas de saúde e a alta condensação populacional, foram motivos imprescindíveis para esclarecer a contingência da dengue. Esse padrão de repetição sociável, associada à negativa flexibilidade de atividades de saneamento ambiental em parte e em características pertinentes, transforma esses espaços favoráveis ao desempenho de propagação da doença.

A precariedade na oferta dessas atividades, especialmente quanto ao fornecimento de água, é capaz de transportar ao acolhimento de hábitos de estoque em recipientes, que por sua parte, podem destacar-se como lugares com potencial de proliferação do vetor (Pedro et al., 2009, p.1937).

Para Kikuti et al., (2015, p.12), essas relações conseguem clarificar o favoritismo ecológico do vetor do mosquito, que acha lugares de evolução larval propícia em espaços com infraestrutura de saneamento básico mais instável.

Com base nos conteúdos estudados através de publicações selecionadas, podemos então concluir que a correlação existente entre baixo nível socioeconômico, renda mínima, moradias inadequadas, aglomerações de famílias, densidade domiciliar e a falta de saneamento básico apresentaram relação positiva com a incidência da doença. Também foi evidente que a desigualdade estrutural em que muitas pessoas vivem, facilitam a existência do mosquito Aedes aegypti, proporcionando assim, um ambiente ideal para que a dengue venha se propagar e se proliferar no meio urbano, trazendo riscos e insegurança para a população, já que a saúde coletiva é manifestada de acordo com as condições ali encontradas.

\section{Considerações Finais}

Ao considerar várias condições sociais que transpassa a dengue, esse aprendizado proporcionou o entendimento, procedendo dos conhecimentos obtidos acerca do tema estudado, da precariedade em que muitas pessoas vivem e são submetidas 
a viverem, e que situações arriscadas de bem-estar público será capaz de levar a um cenário varado por local sem limpeza, com capacidade para a reprodução do intermediário da dengue.

Durante a realização da revisão sistemática, nos deparamos com vários limites: as bases de dados não trouxeram um retorno significativo no primeiro momento, o período de busca e as palavras-chave contiveram os resultados retornados, limitando a procura por publicações. Tivemos também várias dificuldades no que se refere a busca por conteúdos que trouxessem clareza no assunto, segurança e confiabilidade das informações.

Diante do estudo realizado e apresentado, tem como finalidade alcançar a compreensão e tornar a informação ainda mais aproximável das pessoas no que se refere a gravidade da dengue. O estudo busca contribuir para a literatura no tocante à conscientização dos leitores na seriedade que a doença traz à vida dos seres humanos.

Isto posto, os resultados alcançados nesta revisão, levando em consideração os objetivos e as perguntas de procura que foram propostas, auxiliam no suporte para confirmar que necessitam de mais estudos dentro do âmbito da dengue, com o propósito de contribuição para a educação e conhecimento para a população. Recomendam-se como trabalhos posteriores, o estudo de indicadores sociais em pequenas cidades de interior, no intuito de complementar o conhecimento já existente com regiões ainda não descobertas que são de riscos no território brasileiro.

\section{Agradecimentos}

Ao CNPq, a CAPES, a UEPB, ao Coordenador Wellington Candeia de Araújo, ao Professor Francisco Anderson Mariano da Silva e a minha família.

\section{Referências}

Acselrad, H., Mello, C. C. A. \& Bezerra, G. A. N. (2008). O que é justiça ambiental. Garamond. p.78.

Almeida, L. S., Cota, A. L. S. \& Rodrigues, D. F. (2020). Sanitation, Arboviruses, and environmental Determinants of Disease: impacts on urban health. Ciencia \& Saude Coletiva, 25, 3857.

Alves, M. Dos S., Araújo, W. C., Silva, F. A. M. Da. (2021). Analysis on the preponderance that structural inequality causes in health and in the frequency of dengue cases. Research, Society and Development, 10, e12910716426. 10.33448/rsd-v10i7.16426.

Araújo, D. C., Santos, A. D., Lima, S. V. M. A., Vaez, A. C., Cunha, J. O. \& Araújo, K. C. G. M. (2020). Determining the association between dengue and social inequality factors in north-eastern Brazil: A spatial modelling. Geospatial Health, 15.

Barcellos, C. C., Sabroza, P. C., Peiter, P. \& Rojas, L. I. (2002). Spatial Organization, Health and Quality of Life: Use of Spatial Analysis and Indicators in Health Situation Analysis. Informe Epidemiológico do SUS, 130.

Brasil. (1988). Constituição da República Federativa do Brasil de 1988. http://www.planalto.gov.br/ccivil_03/Constituicao/Emendas/Emc/emc90.htm.

Brasil. (2021). IBGE - Instituto Brasileiro De Geografia E Estatística. Síntese de Indicadores Sociais - SIS. https://www.ibge.gov.br/estatisticas/multidominio/c ondicoes-de-vida-desigualdade-e-pobreza/9221-sintese-de-indicadores-sociais.html?=\&t=resultados.

Brasil. (2021). IPEA - Instituto De Pesquisa Econômica Aplicada. Desafios do desenvolvimento. http://desafios.ipea.gov.br/index.php?option=com _content\&view=article\&id=2261:edicao-no-51\&catid=1\&Itemid=5.

Caiaffa, W. T., Assunção, R. M., Mattos, A. M. C. \& Proietti, F. A. (2007). Spatial vulnerability to dengue in a Brasilian urban area during a 7-year surveillance. Journal of Urban Health, 84, 341.

Carabali, M., Harper, S., Neto, A. L., Sousa, G. S., Caprara, A., Restrepo, B. N. \& Kaufman, J. S. (2020). Spatiotemporal distribution and socioeconomic disparities of dengue, chikungunya and Zika in two Latin American cities from 2007 to 2017.

Carmo, R. F., Silva, J. V. J. J, Pastor, A. F. \& Souza, C. D. F. (2020). Spatiotemporal dynamics, risk areas and social determinants of dengue in Northeastern Brazil, 2014-2017: an ecological study. Infectious Diseases of Poverty, 9.

Follador, K., Prado, G. P., Passos, M. G. \& Nothaft, S. C. (2015). Saneamento básico: meio ambiente e saúde. Revista Uningá, $23,24$.

Freitas, M. A., Mattos, A. T. R., Gomes, W. Z. \& Caccia-Bava, M. C. G. G. (2017). Who are they, what do they talk about and who listens to the poor? Ciência \& Saúde Coletiva, 22, 3859-3882.

Gomes, M. P. M., Matos, J. T. Q., Gomes, J. \& Heller, L. (2020). Gestão adequada de resíduos sólidos como fator de proteção na ocorrência da dengue. Revista Panamericana de Salud Publica, 44. 
Gubler, D. J. (1998). Dengue and dengue hemorrhagic fever. Clinical Microbiology Reviews. 482.

Hulley, S. B., Cummings, S. R., Browner, W. S., Grady, D. G. \& Newman, T. B. (2008). Delineando a pesquisa clínica: uma abordagem epidemiológica. (3a ed.), Artmed. p.21.

Kikuti, M., Cunha, G. M., Paploski, I. A. D., Kasper, A. M., Silva, M. M. O., Tavares, A. S., Cruz, J. S., Queiroz, T. L., Rodrigues, M. S., Santana, P. M., Lima, H. C. A. V., Calcagno, J., Takahashi, D., Gonçalves, A. H. O., Araújo, J. M. G., Gauthier, K., Diuk-Wasser, M. A., Kitron, U., Ko, A. L., Reis, M. G., Ribeiro, G. S. B. \& Christopher, M. (2015). Spatial Distribution of Dengue in a Brazilian Urban Slum Setting: Role of Socioeconomic Gradient in Disease Risk. Vol.9.

Londe, L. R., Moura, L. G., Coutinho, M. P., Marchezini, V. \& Soriano, E. (2018). Vulnerability, health and disasters in São Paulo coast (Brazil): Challenges for a sustainable development. Ambiente e Sociedade, 21.

Linde, K. \& Willich, S. N. (2002). How objective are systematic reviews? Differences between reviews on complementary medicine. Journal of the Royal Society of Medicine, p.17.

Machado, J. P., Oliveira, R. M. \& Souza-Santos, R. (2009). Análise espacial da ocorrência de dengue e condições de vida na cidade de Nova Iguaçu. Cad. Saúde Pública, 25, 1025-1034.

Mendonça, F. A., Souza, A. V. \& Dutra, D. A. (2009). Saúde pública, urbanização e dengue no Brasil. Sociedade \& Natureza, 21, 257-269. http://www.seer.ufu.br/index.php/sociedadenatureza/article/view/9606/5783.

MINISTÉRIO DA SAÚDE. (2021). Dengue. https://www.gov.br/saude/pt-br/assuntos/saude-de-a-a-z-1/d/dengue.

Olive, M., Baldet, T., Devillers, J., Fite, J., Paty, M., Paupy, C., Quenel, P., Quillery, E., Raude, J., Stahl, J., Thiann-Bo-Morel, M. \& Roiz, D. (2020). The COVID-19 pandemic should not jeopardize dengue control. PLoS Neglected Tropical Diseases, 14.

Oliveira, K. K. F., Caprara, A. (2019). The social face of Aedes control: women take the floor in a suburban district of Fortaleza, Brazil. Ciência \& Saúde Coletiva, 24, 2983.

Paixão, E. S., Costa, M. C. N., Rodrigues, L. C., Rasella, D., Cardim, L. L., Brasileiro, A. C. \& Teixeira, M. G. L. C. (2015). Trends and factors associated with dengue mortality and fatality in Brazil. Revista da Sociedade Brasileira de Medicina Tropical, 48, 399-405.

Pedro, A. S., Souza-Santos, R., Sabroza, P. C. \& Oliveira, R. M. (2009). Condições particulares de produção e reprodução da dengue em nível local: estudo de Itaipu, Região Oceânica de Niterói, Rio de Janeiro, Brasil. Cad. Saúde Pública. 1937.

Ribeiro, H. \& Vargas, H. C. (2015). Urbanização, globalização e saúde. Revista USP.

Santos, J. P. C., Honório, N. A., Barcellos, C. \& Nobre, A. A. (2020). A Perspective on Inhabited Urban Space: Land Use and Occupation, Heat Islands, and Precarious Urbanization as Determinants of Territorial Receptivity to Dengue in the City of Rio De Janeiro. International Journal of Environmental Research and Public Health, Vol.17, p.6537.

Sousa, C. D. S. S., Sousa, S. C. S. \& Alvares, S. M. (2015). Diretrizes normativas para o saneamento básico no Brasil. Caderno de Geografia, 25(43).

Tauil, P. L. (2001). Urbanização e ecologia do dengue. Cad. Saúde Pública. 17, 99-102.

Tauzer, E., Borbor-Cordova, M. J., Mendoza, J., De La Cuadra, T., Cunalata, J. \& Stewart-Ibarra, A. M. (2019). A participatory community case study of periurban coastal flood vulnerability in southern Ecuador. PLOS ONE, 14.

Teurlai, M., Menkès, C. E., Cavarero, V., Degallier, N., Descloux, E., Grangeon, J., Guillaumot, L., Libourel, T., Lucio, P. S., Mathieu-Daudé, F. \& Mangeas, M. (2015). Socio-economic and Climate Factors Associated with Dengue Fever Spatial Heterogeneity: A Worked Example in New Caledonia. 9.

Whiteman, A., Gomez, C., Rovira, J., Chen, G., Mcmillan, W. \& Loaiza, J. (2019). Aedes Mosquito Infestation in Socioeconomically Contrasting Neighborhoods of Panama City. EcoHealth, 16, 210-221. 